Z Herz-Thorax- Gefäßchir 2011 · 25:329-330

DOI 10.1007/s00398-011-0882-6

Online publiziert: 25. November 2011

(c) Springer-Verlag 2011

\author{
Jochen Cremer \\ Klinik für Herz- und Gefäßchirurgie, Universitätsklinikum \\ Schleswig-Holstein, Campus Kiel
}

\title{
Die Syntax der Koronarrevaskularisation
}

\section{Vier Jahre später}

Liebe Leserinnen und Leser,

die Syntax-Studie hat sich zu einer epochalen Untersuchung der Koronarrevaskularisation mit ganz bemerkenswerten Ergebnissen entwickelt, die von der Datenlage der bisherigen „randomized clinical trials" (RCT) in entscheidenden Punkten abweicht. Dies ist zweifellos wesentlich auf ein grundsätzlich anderes Studiendesign zurückzuführen: die Syntax-Studie intendierte, die Behandlungsrealität besser wiederzugeben und diesem Anliegen wurde mit einem All-comers-Design Rechnung getragen. Grundsätzlich anders gelagert waren aber auch die Entscheidungs- und Strategiewege im Heart-team-Konzept; erstmals erfasste man die Komplexität der koronaren Herzkrankheit systematisch, auch wenn der sogenannte Syntax-Score nicht prinzipiell seiner Gewichtung nach validiert wurde, sondern eher auf den praktischen Erwägungen einer terzilen Betrachtungsweise basiert. So ist es dann auch nicht verwunderlich, dass die Syntax-Studie in ihrer Ausrichtung auf eine praxisnahe Behandlungsrealität die Ergebnislage von artifiziellen RCTs mit einer Einschlussrate von $3-7 \%$ der gescreenten Patienten mit der Ergebnissituation einer Registeranalyse verbindet, wie beispielsweise des New York Heart Registry's, über das in den verschiedenen Hannan-Publikationen ausführlich informiert wurde.

Für diejenigen von Ihnen, die nicht die Gelegenheit hatten, den Sonntagmorgen des 2. Oktober 2011 beim Annual Meeting der EACTS in Lissabon verbringen zu können, sei berichtet, dass Patrick Serruys als kardiologischer Principal Investigator die 4-Jahres-Ergebnisse des Gesamtkollektivs der randomisierten Patienten detailliert darstellte.

\section{In general persisting positive trends for surgery?}

Es konnte jedenfalls erstmals eine signifikant bessere Überlebensrate für das chirurgische Gesamtkollektiv mit einer Gesamtmortalität von $8,8 \%$ für die Chirurgie im Vergleich zu 11,7\% für PCI konstatiert werden. Hier sei daran erinnert, dass die Überlebensraten im gesamten Verlauf, also insbesondere auch in der Frühphase in den ersten 30 Tagen, in der Chirurgie ungeachtet aller Invasivität günstiger sind als für die Intervention, sowohl in der Betrachtung der Gesamtmortalität als auch des Herztods. Mit Blick auf den Herztod ist die Differenz nach vier Jahren sogar noch evidenter: 7,6\% Sterblichkeit bei PCI vs. 4,3\% Sterblichkeit bei CABG.

Darüber hinaus haben sich auch die signifikanten Unterschiede hinsichtlich des Auftretens myokardialer Infarkte und auch des Bedarfs für eine erneute Revaskularisation („repeat revascularization“) bestätigt, während die Inzidenz von Schlaganfällen nach vier Jahren keine wesentlich verschiedenen Ergebnisse zeitigt. Für den Patienten eine glänzende Perspektive: besseres Überleben mit weniger Infarkten, einem stabileren Ergebnis und erhöhter Sicherheit.

In der Subgruppenanalyse mit niedrigen, intermediären und hohen ScoreWerten hinsichtlich der Komplexität der
KHK - und hierbei werden per definitionem Gruppen von jeweils etwa $300 \mathrm{~Pa}$ tienten miteinander verglichen - findet sich dann im Low-score-Bereich lediglich ein signifikanter Vorteil hinsichtlich des Bedarfs für eine erneute Revaskularisationen, während es schon bei intermediärer Komplexität zu signifikant mehr Infarkten nach PCI (9 vs. 3,6\%) kommt; im hochkomplexen Bereich - vielfach die Alltagssituation für den Chirurgen - zeigt sich gar ein dramatischer, 8-\%iger Unterschied in der Todesrate (PCI 16,1\%, CABG $8,4 \%$ ) ebenfalls verbunden mit weniger Infarkten und weniger Bedarf für erneute Revaskularisation.

Die Frage an den Patienten lautet: Wollen Sie sich lieber einer sicheren, hochpräzisen Bypassoperation unterziehen, die Ihnen ein langes Überleben mit hoher Lebensqualität und weitgehender Beschwerdefreiheit ermöglicht oder ziehen Sie einen eleganten, weniger invasiven Eingriff vor, der jedoch in Ergebnisqualität und Nachhaltigkeit der Bypassoperation nicht das Wasser reichen kann?

Meine sehr verehrten Damen und Herren Leser, Sie sehen, die Diskussion bleibt spannend und Herzchirurgen sollten unbedingt die aktuelle Datenlage zur Koronarrevaskularisation kennen, auch um die Basis aktueller Leitlinien präsent zu haben. Diese Leitlinien wiederum sind Pflichtlektüre für jeden herzchirurgisch tätigen Arzt; hier wird momentan an der Anpassung der Europäischen Guideline auf deutsche Verhältnisse (Nationale Versorgungsleitlinie „Chronische koronare Herzkrankheit“) gearbeitet, um in der 
kollegialen Heart-team-Diskussion bestens argumentieren zu können.

Auch im Namen der anderen Herausgeber wünschen ich Ihnen viel Spaß bei der Lektüre der aktuellen Ausgabe, auch wenn Sie in ihr zur neuen Datenlage der Syntax-Studie noch keinen Artikel finden.

Ihr

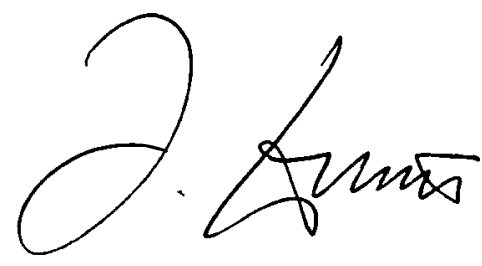

Prof. Dr. Jochen Cremer

\section{Korrespondenzadresse}

\section{Prof. Dr. Jochen Cremer}

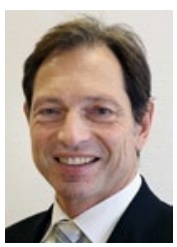

Klinik für Herz- und

Gefäßchirurgie, Universitäts-

klinikum Schleswig-Holstein,

Campus Kiel

Arnold-Heller-Straße

324105 Kiel

JCremer@kielheart.uni-kiel.de

\section{Galenus-von-}

Pergamon-Preis 2011

\section{Springer Medizin zeichnet exzellente Forschung und ehrenamtliches Engagement aus}

Auch in diesem Jahr hat Springer Medizin den von der Ärzte Zeitung Verlags $\mathrm{GmbH}$ gestifteten Galenus-von-Pergamon-Preis vergeben und würdigt damit exzellente Forschung in Deutschland. 12 unabhängige Experten haben im Oktober 2011 über die Preisträger in den Kategorien Primary Care, Specialist Care und Grundlagenforschung entschieden. Als Schirmherrin des Galenus-von-PergamonPreises lobte die Bundesministerin für Bildung und Forschung, Frau Professor Dr. Annette Schavan, die herausragenden Leistungen aller Forscher-Teams.

Primary Care

Der Preis in der Kategorie Primary Care würdigt ein Medikament, das bei einer breiten Patientengruppe eingesetzt wird. In diesem Jahr hat Amgen/GlaxoSmithKline (GSK) den Preis für Prolia ${ }^{\circledast}$ erhalten. Mit Prolia $^{\circledR}$ steht erstmals ein monoklonaler Antikörper zur gezielten Osteoporosetherapie zur Verfügung. Der Antiköper hemmt die Knochenresorption und schützt somit Frauen in der Postmenopause und Männer mit Prostatakrebs vor Frakturen.

\section{Specialist Care}

Der Preis in der Kategorie Specialist Care zeichnet ein Medikament aus, das zur Behandlung seltener Erkrankungen verwendet wird. In diesem Jahr ist Amgen der Gewinner für Nplate ${ }^{\circledR}$, das erste zugelassene Medikament zur Stimulierung der Thrombozytenbildung. Es ist indiziert zur Behandlung von Erwachsenen mit einer chronischen Immun-(idiopathischen)thrombozytopenischen Purpura (ITP), die auf andere Therapien nicht ansprechen.
Die Preisträger in diesen beiden Kategorien erhielten jeweils eine Medaille und eine Urkunde.

Grundlagenforschung

In dieser Kategorie wird ein Bewerber prämiert, der eine herausragende wissenschaftliche Arbeit in der pharmakologischen Grundlagenforschung eingereicht hat. Die Auszeichnung ging an Professor Wolfgang Kühn aus Freiburg. Er und sein Team haben sich in der Forschung um die Entschlüsselung molekularer Mechanismen der autosomal dominanten polyzystischen Nierenerkrankung (ADPKD) verdient gemacht. Der Preisträger erhält zur Medaille und Urkunde zusätzlich ein Preisgeld in Höhe von 10.000 Euro.

CharityAward 2011

Mit dem im Jahr 2009 erstmals gestifteten CharityAward zeichnet Springer Medizin jährlich Menschen und Organisationen aus, die sich ehrenamtlich um behinderte, kranke und hilfsbedürftige Menschen verdient gemacht haben. Wer Gewinner wird, entscheiden die Leser der Medien von Springer Medizin. Preisträgerin 2011 ist die in Peru geborene Ärztin Jenny De la Torre: Sie wird für ihren inzwischen 16-jährigen Einsatz für Obdachlose in Berlin-Mitte ausgezeichnet. Im Rahmen dieses Engagements hat sie eine Stiftung gegründet und das Berliner "Gesundheitszentrum für Obdachlose" aufgebaut. Der Award besteht aus einem Medienpaket in einem Wert von 100.000 Euro und einem Barscheck über 50.000 Euro. Schirmherr des Preises ist Bundesgesundheitsminister Daniel Bahr. 